\title{
Learning a Potential Function From a Trajectory
}

\author{
David R. Brillinger
}

\begin{abstract}
This ]elter concerns the use of stochastic gratient syslenus in the modelings of the paths of noving particles and the consecjuent estinustion of a pofentual lunction. The work proceteds by setling dow a paramul ric or nonparanıt ric model for the potenlial funclion. The muthod is sinoplt, direct, and fluxible, being' hasted on a lineitr nodtal and the least sipuit res. Explanatories, altractors, ind repellors may bi included directly. The large sample distribution of the estinated potential funclion is provided, under speetfic assumptisns. Thire are dired extensions to updating, sliding window, adaptive, robust, and real-lime variants. An example anilyring the path of an th is prestegled.
\end{abstract}

Index Terms-Mobility modul, moniloring, poltential function, stochastic djffurential Equation, stochustic gradjeng system.

\section{INTRODUCTION}

L OCATION signals of moving objects. abtaned tor example by GPS or LORAN, have become common in practice. Typicully, one has sedtered positions along trajectories of the objects. The questions of how to summarize, how to predict, and how to simulate such movements arise. This happens particularly when n number of paths are involved or the path of an object is a tangle. See Fig. 1, which shows 157। Iocitions bver a period of a month along the track of an clk in Starkey Project in Oregon. (Rererence $[1]$ prowides the project's website address.)

This letter provides a unitied approuch for dealing with movement modeling and associated data. The fields in which movement data have arisen include animal track ing [2], [3] and socee [4]. There are papers developing a stalistical potential approach io tracks. These include [2], [3], and references therein. This letter provides some formal buekground missing in those papers, discussion. and an example.

Let $r$ denote a point in $I^{\prime 2}$. (In the mathematical expressions below, all the vectors appearing are colume vectors and sel in buldfice.) A polential function, $V(r)$, is a real-valued function of locution. Its use cun lead to simpler representations of moion than those based on modeliug velocities directly. One can note that in the owerdamped case, the equation of motion of a particle in the potential licld, $V(r)$, is

$$
d r(f)=-F V(r(t)) d t
$$

having assumed $Y(r)$ dirlerentiable and with $\nabla$ denoting the gradient. |The negative sign in (1) is traditional.] The entity dr(t) $/$ df is called a vector tield. When $y=2$. the level surfaces

Manuscripl rectived Devember t2. 2006: rewised April 2. 2007. Thu associallo edilor covodinating the review of this manuseript and apperoving it for publicat tion wisk Dr. B Eian M. Siddler.

The auther is with the Stallisties Depurtmont. Unirersity of Califormis Berkeley, Berkeley, CA y472tI USA (e-mail: brillogstat herkeley.edu).

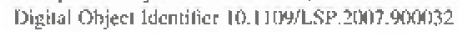

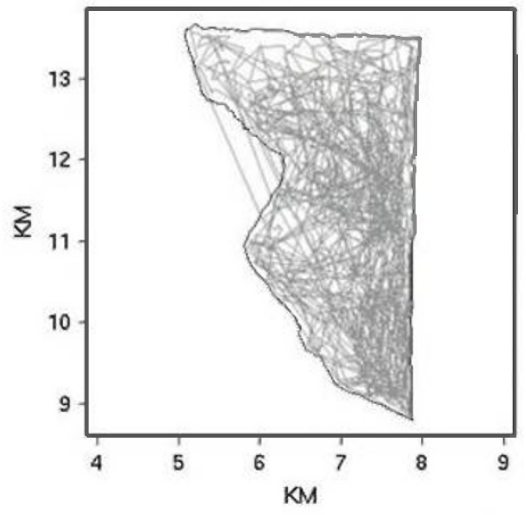

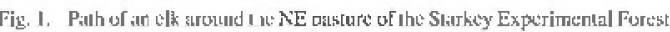
in Oregon. Localions were cstimated approximately cyery two hours and ase joined by consecutiwe straigh lines.

of the potcntial function are convenchtly displayed in contour form and its gradient as arrows on a grid (see Figs. 2 and 3 )

The estimation method to he presented can he motivated hy stochastic gradient systems, that is, systems that can be written in the time invariant case as

$$
d r(t)=-F V(r(t)) d t+\sigma(r(t)) d W(t)
$$

for some differentrable $f$ with $B(t)$ a $j$-dimensional Brownian motion and a a $\mu$ by $\psi$ malrix. Expression (2) is a parlicular case of the stochastic differential equation (SDE)

$$
d r(t)=\mu(\tau(t)) d t+\sigma(r(t)) d B(t)
$$

What dislinguishes the tratlitional SDE work from the present sludy is that the drift term $\mu$ here has the special form $-\nabla V$ for some real-valued function $F$. It will be seen that the modeling situation is simplified when such a $V$ is assumed to exist.

\section{I1. PROTILIM AND APPROACH}

The basic problem assumes the model (2) and seeks to learn $V(r)$ given dala $\left(t_{j}, r\left(t_{i}\right), \dot{x}=1, \cdots, \pi\right)$. These data will be viewed ats locations at successive limes, $\left\{t_{i}\right\}$, or an objoct moxing along a trajectory of the process (2). One seeks both vector lield and potential function estimates.

Supposing that $\nabla V(r)$ is a sinooth function of $r$, and that the observation times are close together, one can sel down the following approximation lo (2):

$$
\begin{aligned}
r\left(t_{i+1}\right)-r\left(t_{i}\right)=-\Gamma V\left(r\left(t_{i}\right)\right)\left(t_{i+1}-t_{i}\right) & \\
& +\left(t_{i+1}-t_{+}\right)^{1 / 2} \sigma Z_{i+1}
\end{aligned}
$$

$1070-990 \mathrm{k} \$ 250002007$ IEFH: 


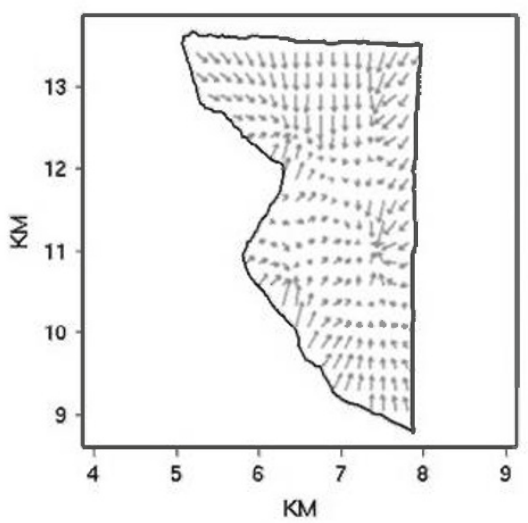

Fig. 2. Estimated vector field for the path of Fig. 1.

for $\dot{x}=1,2,4, \cdots, n$, with $\sigma$ a $p$ by $p$ matrix and with the $Z$, independent ${ }^{\mu}$-dimensional variates having mean 0 and covariance matrix $I$. The reason for the multiplier $\left(t_{i+1}-l_{j}\right)^{1 / 2}$ is that for real-yalued Brownian, Var $(d B(t))=d t$.

The approximation

$$
\begin{array}{r}
\left(\tau\left(t_{i+1}\right)-\tau\left(t_{i}\right)\right) /\left(t_{i+1}-t_{i}\right) \\
=\mu\left(r\left(t_{i}\right)\right)+\sigma Z_{i+1} /\left(t_{i+1}-t_{i}\right)^{1 / 2}
\end{array}
$$

for the SDE (3) was employed in [2] and [3] for elk and movement and is employed in the example of this letter. In [2], an carly attempt was made at estimating a potential function by numerical inlegration and simulation. The question was asked whether the vector lield, $\boldsymbol{\mu}$. had the form $-\nabla V(r)$. This may he siludied hy comparing an unrestricted estimale of $\mu$ with one assuming the existence of a polential function. The approach of papers [2] and [3] was informal.

\section{Potential Funictions}

A basic issue is how to dewcribe matlematically a potencial function, $V^{\prime} \mathbf{r}, \mathbf{r}$ in $l \%^{\prime}$. Suppose one exists. For introductory purposes in the develapment, suppose $I$ is linear in a vector valued parameer $\beta$. Write $\mathfrak{I}(\boldsymbol{r})=\phi(\boldsymbol{r})^{T} \beta$ with $\phi$ am $L$ by I vector of functions of known form and $\beta$ an $L$ by I unknown parameter. Examples of such a $\mathrm{I}$ follow. The gratient of $V$ is the $p$ by 1 vectur $\Gamma \phi i r i{ }^{\prime}$ it

Example 1: Polynomial expansion.

Consider $V(r)=\sum \beta \ldots r^{m}$. where $m=\left(m_{1}, 1+\cdots, m_{p}\right)$ and

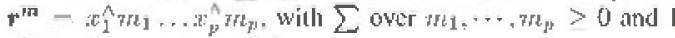
$<m h_{1}+\cdots+m h_{j}<M$.

One could umploy a trigonometric polynomial, a spline function, ог is wavelet expansion here. Many funclions, $V$, can be well approximated by taking $M$ large. In practice, one might employ $M_{n}$ with $M_{n}$ increasing with $\pi t$.

Example 2: Nodte based.

Consider nodal points $u_{r}, \bar{l}=1, \cdots, L$ in $R^{p}$ and $\operatorname{set} V(r)=$ $\sum A_{1} h\left(r-n_{i}\right)$ for some real-walued differentiable kemel $K$.

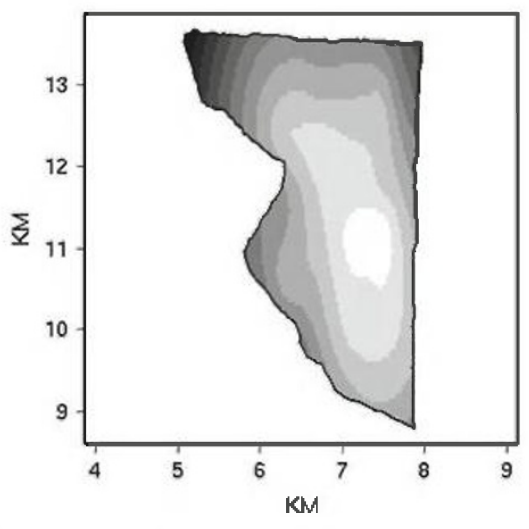

Fig. 3. Estimated potenlial tor the patth of Fig. I. The lighler shading : ")lln"

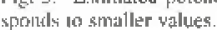

As a spectic example of $K$, one has the radial basis thin plate splines [5. pp. 30-34]

$$
K(r)=|r|^{2 q-\gamma /} \log |r| f \text { for } p \text { even, }=|r|^{2 q-r}-\text { for } w \text { odd. }
$$

Here if denotes the order of differentiability of $k, 2 f-p>0$. and $|\mathbf{r}|=\left(\boldsymbol{r}^{T} \boldsymbol{r}\right)^{1 / 2}$. An expression like (6) leads to a smooth representation for $V$.

Example 3: Attraction and repulsion.

Consider a region $A$ and a point $\mathbf{r}$ outside $A$. Potential funclions can be set down leading to altraction or repulsion from L. Specifically, if one lets it a $r$. denote the minimum distance

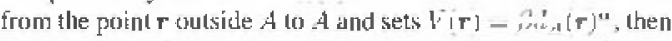
for $n>0$. one has attruction to $A$ and repulsion if $\alpha<0$. One can rewerse attraction and repulsion by changing the sign of $d_{4}$. It can be convenient to use $V(r)=\beta_{1} \log d_{-4}(r)+\beta_{2} d_{-4}(r)$ for similä purposes.

The functional forms of Exumples I-3 may be added together to prowide other forms.

Reference |6| considers the observed trajectory of a monk stal near the island of Molokai employing the mixed function

$$
V(r)=\gamma_{1} x+\gamma_{2} y+\gamma_{11} x^{2}+\gamma_{12}\left(x y+\gamma_{22} y^{2}+\zeta / d[(x, y)(7)\right.
$$

where $\mathbf{r}-(r . y)^{S}$ is in $R^{2}$ and represents the Iscalion of the animal on the ocean surface. The value $d(x . y)$ is the distance from the localion $r$ to the nearest point on the island. The $r$ 's and $C$ are unknown parameters to be estinleted. The linal term in (7) keeps the seal off of the island. A ditiferent monk seal is sudued in $|7|$, and a diflerent, now time dependent, potential function is employed

$$
V(r, l)=\alpha \log d(r, l)+\beta d(r, b)
$$

with $d(r$. t) being the animal's distance from an attractor at lime t. The allractor swilched depending on whether the animal wes on an outbound or an irbound joumey. 
Reference [4] studies the motion of a soccer ball during a very exciting World Cup moment. The potential function used is

$$
\alpha \log d(\boldsymbol{r})+\beta d(\boldsymbol{r})+\gamma_{1} x+\gamma_{2} y+\gamma_{11} x^{2}+\gamma_{12} x y+\gamma_{22} y^{2}
$$

with $d(\boldsymbol{r})$ the shortest distance to the goalmouth from $\boldsymbol{r}=(x, y)^{T}$. The first two terms lead to attraction to the goalmouth and the remaining to general motion on the field.

Potential function and vector field estimates are provided in each of the papers just referenced. The function $V(\boldsymbol{r})$ is linear in the parameter, and least squares is employed as the estimation procedure in each case. The function could be nonlinear, and then, nonlinear least squares could be employed. Reference [8] develops asymptotic results pertinent to the nonlinear case. $\mathrm{Al}$ ternately, the $\left\{\boldsymbol{Z}_{i}\right\}$ in (5) could be non-Gaussian and maximum likelihood estimation employed.

\section{ESTIMATION}

The representation (4) with $\boldsymbol{r}$ in $R^{p}$ and $\nabla V(\boldsymbol{r})=\nabla \boldsymbol{\phi}\left(\boldsymbol{r}_{i}\right)^{\bar{T}} \boldsymbol{\beta}$ will be employed. The values $\boldsymbol{r}\left(t_{i}\right)$ will be written $\boldsymbol{r}_{i}$. Consider the $p$ by 1 vector $\left(\boldsymbol{r}_{i+1}-\boldsymbol{r}_{i}\right) /\left(t_{i+1}-t_{i}\right)^{1 / 2}$. Following expression (5), the model has the form

$\left.\left(\boldsymbol{r}_{i+1}-\boldsymbol{r}_{i}\right) /\left(t_{i+1}-t_{i}\right)^{1 / 2}=-\nabla \phi\left(\boldsymbol{r}_{i}\right)^{T} \beta\left(t_{i+1}-t_{i}\right)^{1 / 2}+\sigma \boldsymbol{Z}_{i+1}\right)$

$i=1, \cdots, n-1$ involving the $L$ by 1 vector $\boldsymbol{\beta}$, the $L$ by $p$ matrix $\nabla \boldsymbol{\varphi}\left(\boldsymbol{r}_{i}\right)$, the $p$ by $p$ matrix $\boldsymbol{\sigma}$, and the $p$ by 1 vector $\boldsymbol{Z}_{i+1}$ Suppose $\sigma=\sigma \boldsymbol{I}$ with $\sigma$ positive and $\boldsymbol{I}$ the $p$ by $p$ identity matrix. Stack the $n-1$ values $\left(\boldsymbol{r}_{i+1}-\boldsymbol{r}_{i}\right) j^{\prime}\left(t_{i+1}-i_{i}\right)^{1 / 2}, i=1, \cdots, n-1$ vertically to form the $(n-1) p$ by 1 array $\boldsymbol{Y}_{n}$. Stack the $n-1$ matrices $-\nabla \varphi\left(\boldsymbol{r}_{i}\right)^{T}\left(t_{i+1}-t_{i}\right)^{1 / 2}$ to form the $(n-1) p$ by $L$ matrix $\boldsymbol{X}_{n}$. Stack the $n-1$ values $\sigma \boldsymbol{Z}_{i+1}$ to form $\boldsymbol{\varepsilon}_{n}$. Then one has the regression model

$$
\boldsymbol{Y}_{n}=\boldsymbol{X}_{n} \boldsymbol{\beta}+\varepsilon_{n}
$$

with the difference from ordinary regresssion that $\boldsymbol{Y}_{n}$ and $\boldsymbol{X}_{n}$ are statistically dependent. Using a generalized inverse, if necessary, one can compute an ordinary least-squares estimate $\boldsymbol{b}=\left(\boldsymbol{X}_{n}^{T} \boldsymbol{X}_{n}\right)^{-1} \boldsymbol{X}_{n}^{\boldsymbol{T}} \boldsymbol{Y}_{n}$ of $\boldsymbol{\beta}$, and then, if $\boldsymbol{\varphi}(\boldsymbol{r})^{T} \boldsymbol{\beta}$ is estimable, $\varphi(\boldsymbol{r})^{T} \boldsymbol{b}$ is a reasonable estimate of $V(\boldsymbol{r})$.

Supposing the individual entries of $\varepsilon_{n}$ to be independent, zero mean, variance $\sigma^{2}$ variates, asymptotic properties of $\varphi(r)^{T}$ may be obtained from [9, Theorem 3]. The theorem is given in the Appendix.

Let $y_{j}$ denote the $j$ th row of $\boldsymbol{Y}_{n}$. Let $\boldsymbol{x}_{j}^{T}$ denote the $j$ th row of $\boldsymbol{X}_{n}^{T}$. One can compute $s_{n}^{2}=((n-1) p)^{-1} \sum\left(\boldsymbol{y}_{j}-\boldsymbol{x}_{j}^{T} \boldsymbol{b}\right)^{T}\left(\boldsymbol{y}_{j}-\right.$ $\left.\boldsymbol{x}_{j}^{T} \boldsymbol{b}\right)$ as an estimate of $\sigma^{2}$ and, for example, set down a confidence interval for $\boldsymbol{\varphi}(\boldsymbol{r})^{T} \boldsymbol{\beta}$ using the results of [9]. Specifically, provided $\lim \log \lambda_{\max }\left(\boldsymbol{X}_{n}^{T} \boldsymbol{X}_{n}\right) / n \rightarrow 0$ almost surely, one has $s_{n} \rightarrow \sigma$ almost surely and by a Slutsky Theorem

$$
\left(\boldsymbol{\varphi}(\boldsymbol{r})^{T}\left(\boldsymbol{X}_{n}^{T} \boldsymbol{X}_{n}\right)^{-1} \boldsymbol{\varphi}(\boldsymbol{r})\right)^{-1 / 2} \boldsymbol{\varphi}(\boldsymbol{r})^{T}(\boldsymbol{b}-\boldsymbol{\beta}) / s_{n} \rightarrow N(0,1)
$$

with $N(0,1)$ the standard normal. This leads to the approximate $100(1-\alpha) \%$ confidence interval

$$
\varphi(\boldsymbol{r})^{T} \boldsymbol{\beta}=\varphi(\boldsymbol{r})^{T} \boldsymbol{b} \pm z_{\alpha / 2}\left(\boldsymbol{\varphi}(\boldsymbol{r})\left(\boldsymbol{X}_{n}^{T} \boldsymbol{X}_{n}\right)^{-1} \varphi(\boldsymbol{r})^{T}\right)^{1 / 2} s_{n}
$$

where $z_{\alpha / 2}$ denotes the $100 \alpha / 2$ percent point of the standard normal. As mentioned in [9], one could use the $F$ distribution to construct an approximate confidence region for a collection of values $\left\{\boldsymbol{\phi}\left(\boldsymbol{r}_{k}\right)^{T} \boldsymbol{\beta}\right\}$.

\section{EXAMPLE}

The Starkey Project is a large area in Oregon set aside to study the interactions of elk, deer, cows, and man sharing an environment [1]. Fig. 1 shows a sampled trajectory of one of the elk in the NE Pasture. There were 1571 GPS locations and times of location obtained with a time interval of approximately two hours between successive locations. It is recognized that the theory connecting the sampled times case to the continuous time case expects the times to be close together. It is still anticipated that the discrete model studied is of interest in its own right and will provide results of practical use.

A potential function $V(\boldsymbol{r})$ was approximated by a thin plate radial basis spline employing the kernel function of (6) with $p$ and $q=2$, and $L=36$. The $x$ and $y$ components of the $\boldsymbol{u}_{l}$ were taken to be the $100 \mathrm{~m} / 7, m=1, \cdots, 6$ percentiles of the standardized $x$ and $y$ values. These values were chosen was for illustrative purposes.

The coefficients $\beta_{l}$ were estimated by ordinary least squares employing the model (5) with $\sigma=\sigma \boldsymbol{I}$. The results are provided in Figs. 2 and 3. One sees the confusion of Fig. 1 much reduced. A point of attraction appears near the point $(7.5,11.0)$. When one looks at a topographic plot of elevations, the point of attraction appears to be a valley/canyon of sorts. Fig. 3 provides an image plot of the potential function. Now one sees the point of attraction immediately.

The confusion of Fig. 1 has been referred to. An empirical gradient plot is similarly confused.

\section{EXTENSIONS AND CONCLUSION}

Various generalizations of the letter's results may be mentioned. One could set down an expansion for $V$ employing wavelet functions. One could consider updating methods for real-time work, e.g., those based on a Kalman filter. One could envisage a potential function as a spatial state variable and the paths of objects determined by the measurement equation. If the potential function is changing slowly, one could consider a sliding window estimate [10]. Estimates that are robust to non-normailty and resistant estimation can be considered. In video analysis, one might consider the model $\boldsymbol{I}(\boldsymbol{r}, t)=\boldsymbol{I}_{0}(\boldsymbol{r})+\delta(\boldsymbol{r}(t)-\boldsymbol{r})$ with $t$ indexing the video frames and $\delta$ the Dirac delta. The term $I_{0}$ represents a stationary background and $\boldsymbol{r}(t)$ the location of an object moving around in the scene [11].

This letter presents an estimation method for handling moving objects. The computations may be implemented by the least-squares algorithm. The model may be viewed as parametric or nonparametric. 


\section{APPENDIX}

Because of the statistical dependence of the location of the object at time $t_{i}$ on past locations, one needs special arguments to get the asymptotic distribution. For the simple cases of the letter, results based on martingale arguments are available in [9]. A result is [9, Theorem 3].

Theorem. Consider the regression model $y_{j}=\boldsymbol{x}_{j}^{T} \boldsymbol{\beta}+\varepsilon_{j}, j=$ $1,2, \ldots$ with the $\left\{\varepsilon_{j}\right\}$ martingale differences with respect to an increasing sequence of $\sigma$-fields $\left\{F_{N}\right\}$. Suppose that $\sup _{n} E\left(\left\|\varepsilon_{N}\right\|^{\alpha} \mid F_{N-1}\right)<\infty$ almost surely for some $\alpha>2$. Suppose further that $\lim _{n \rightarrow \infty} \operatorname{var}\left(\varepsilon_{N} \mid F_{N-1}^{\prime}\right)=\sigma^{2}$ almost surely for some nonstochastic $\sigma$. Define $\boldsymbol{X}_{N}=\left[\boldsymbol{x}_{1} \ldots \boldsymbol{x}_{N}\right]^{T}$. Assume that $\boldsymbol{x}_{1}$ is a $\vec{F}_{j-1}$-measurable random variable and that there exists a nonrandom positive definite symmetric $L$ by $L$ matrix $\boldsymbol{B}_{N}$ for which $\boldsymbol{B}_{N}^{-1}\left(\boldsymbol{X}_{N}^{T} \boldsymbol{X}_{N}\right)^{1 / 2} \rightarrow \boldsymbol{I}$, sup $1 \leq i \leq N\left\|\boldsymbol{B}_{N}^{-1} \boldsymbol{x}_{N}\right\| \rightarrow 0$ in probability. Then as $N \rightarrow \infty$

$$
\left(\boldsymbol{X}_{N}^{T} \boldsymbol{X}_{N}\right)^{1 / 2}(\boldsymbol{b}-\boldsymbol{\beta}) \rightarrow N\left(0, \sigma^{2} \boldsymbol{I}\right)
$$

in distribution.

Note that zero-mean independent observations like the successive entries of $\varepsilon_{n}$ of (9) form a martingale difference sequence with respect to the $\sigma$-field generated by the preceding locations.

Reference [15] shows that under the further assumption, lim $\log \lambda_{\max }\left(\boldsymbol{X}_{n}^{T} \boldsymbol{X}_{n}\right) / n \rightarrow 0$ almost surely, one has $s_{n} \rightarrow \sigma$ almost surely.

\section{ACKNOWLEDGMENT}

The author would like to thanks A. A. Ager and H. K. Preisler for involving him with Starkey data sets and ideas that proved useful.

\section{REFERENCES}

[1] Starkey Project. [Online]. Available: http://www.fs.fed.us/pnw/ starkey.

[2] D. R. Brillinger, H. K. Preisler, A. A. Ager, and J. G. Kie, "The use of potential functions in modelling animal movement," in Data Analysis from Statistical Foundations, A. K. M. E. Saleh, Ed. Huntington, NY: Nova, pp. 369-386. [Online]. Available: http://www.stat.berkeley.edu/ $\sim$ brill/Papers/fraser8.pdf, (2001a).

[3] D. R. Brillinger, H. K. Preisler, A. A. Ager, J. Kie, and B. S. Stewart, Modelling Movements of Free-ranging Animals, Univ. California Berkeley Statistics, Tech. Rep. 610. [Online]. Available: http//www stat.berkeley edu/ brill/Preprints/610.pdf, (2001b).

[4] D. R. Brillinger, "A potential function approach to the flow of play in soccer," J. Quant. Anal. Sports vol. 3, no. 1, 2007. [Online]. Available: http://www.stat.berkeley.edu/ brill/Papers/jqas.pdf.

[5] G. Wahba, Spline Models for Observational Data. Philadelphia, PA: SIAM, 1980.

[6] D. R. Brillinger, B. Stewart, and C. Littnan, "A meandering hylje," in Festschrift for Tarmo Pukkila on His 60th Birthday, E. P. Liski, J. Isotalo, S. Puntanen, and G. P. H. Styan, Eds, Dept. Mathematics, Statistics and Philosophy, Univ. Tampere. [Online]. Available: http://www. tics and Philosophy, Univ. Tampere. [Online]. Available: http://www. stat.berkeley.edu/ brill/Papers/hyljel.pdf, 2006

[7] D. R. Brillinger, B. Stewart, and C. Littnan, "Three months journeying of a Hawaiian monk seal," Lecture Notes Statist. [Online]. Available: http://www.stat.berkeley.edu/ brill/Papers/monk33.pdf 2006b.

[8] T. L. Lai, "Asymptotic properties of nonlinear least squares estimates in stochastic regression models," Ann. Statist., vol. 10, pp. 1917-1930, 1994.

[9] T. L. Lai and C. Z. Wei, "Least squares estimation in stochastic regression models with applications to identification and control of dynamic systems," Ann. Statist. vol. 10, pp. 154-166, 1982.

[10] M. Belge and E. L. Miller, "A sliding window RLS-like adaptive algorithm for filtering alpha-stable noise," IEEE Signal Process. Lett., vol. 7, no. 4, pp. 86-89, Apr. 2000

[11] V. Zlokolica, A. Pizurica, and W. Philips, "Noise estimation for video processing based on spatio-temporal gradients," IEEE Signal Process. Lett., vol. 13, no. 6, pp. 337-340, Jun. 2006. 